\title{
Modeling Aventurescent Gems with Procedural Textures
}

\author{
Andrea Weidlich \\ Alexander Wilkie
}
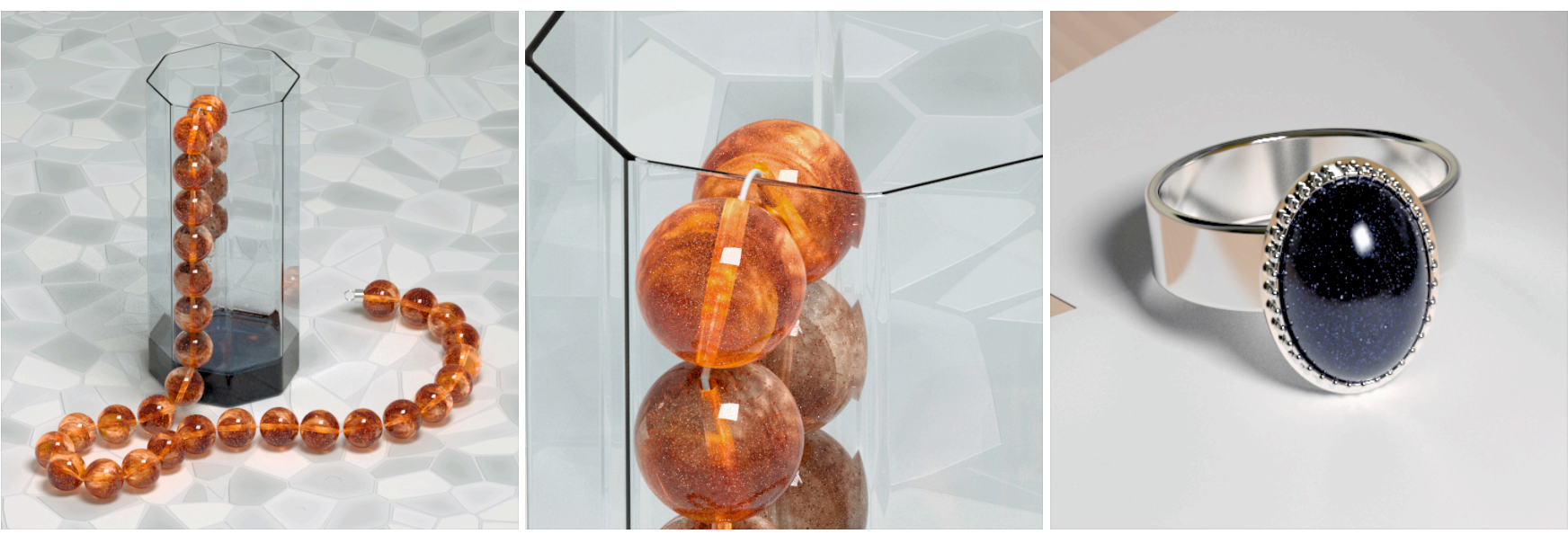

Figure 1: Examples of gemstones that exhibit aventurescence, and that have been rendered using the methods described in this paper. The beads on the necklace that is draped on the drinking glass in the leftmost image are examples of Sunstone, a genuine mineral that is sought after for its ephemeral, amber-like appearance with glittering inclusions. The middle image is a close-up of several beads, while the picture on the right shows a silver ring with a gem made from black aventurescent glass.

\section{Abstract}

In this paper we present the results of an investigation on how one can model aventurescence, an interesting and optically appealing property of some gemstones. Our goal was to find a method that is both efficient and reasonably realistic, and that can still be used in the context of a global illumination rendering system.

CR Categories: I.3.7 [Computer Graphics]: Three-Dimensional Graphics and Realism

Keywords: gemstone rendering, aventurescence, procedural textures

\section{Introduction}

Crystals and minerals can exhibit a large and diverse number of interesting optical properties. One particular feature is that of aventurescence, which is a glittering effect caused by small, highly reflective crystalline metallic inclusions of up to $1 \mathrm{~mm}$ in size embedded in a parent mineral.

These inclusions, which we will refer to as particles in this paper, are commonly copper, Hematite or Mica. The faces of these tiny crystals (for which we use the name particle facets) act as tiny mirrors, so that the otherwise transparent or translucent gemstone sparkles and glitters when turned around. The overall effect is not unlike that of certain types of metallic paint, albeit one with comparatively few - and, by the standards of metallic paint, rather large - metallic flakes embedded in it.

The effect is found in a number of chemically and crystallographically diverse minerals, such as Sunstone, Aventurine Quartz or Goldstone. Due to the visually appealing nature of the effect, minerals with this property are sought after as decorative objects and gemstones.

Especially for large and comparatively valuable raw gemstones like e.g. Sunstone, the cutting and polishing that leads to a finished gem requires careful consideration. A proper cut can greatly increase the value of a stone, and one only gets one attempt at getting this right. Therefore computer-aided prototyping of gemstone cuts - which is something normally associated with "classical" gemstones such as diamond or sapphire - might be potentially a desirable feature even for minerals which exhibit aventurescence.

The aim of this paper is to enhance computer graphics knowledge in the area of gemstone rendering by reporting on our practical experiences in reproducing this particular appearance attribute. We use Aventurine glass, a synthetic substance which exhibits the effect very clearly, to demonstrate our technique, but as discussed in section 5 the method we propose can be adapted to represent a variety of different genuine minerals.

\section{Related Work}

While gemstone rendering is not a large research area within graphics, it has over the years yielded a number of publications about various effects that are peculiar to crystals and precious stones.

Dispersion was first examined by [Thomas 1986] and later by [Yuan et al. 1988]. Further investigation was done by [Sun et al. 2000], who also investigated absorption in homogeneous transparent materials [Sun et al. 1999]. Polarisation was examined by [Wolff and 
Kurlander 1990], later by [Tannenbaum et al. 1994] and [Wilkie et al. 2001]. Recently [Guy and Soler 2004] found a way to render gemstones in realtime.

To the best of our knowledge, nobody so far has investigated aventurescence in gemstones. The closest work to ours is [Ďurikovič 2002; Durikovič et al. 2002], but unlike to our work, this approach explicitly modeled the particles with a triangular mesh. [Ershov et al. 1999; Ershov et al. 2001] introduced a surface model for pearlescent paint that includes sparkling effects based on a statistical approach for static scenes.

Based on these models [Ershov et al. 2004] later showed how to reverse engineer the appearance of real paint by appropriate mapping of the surface parameters of their paint model.

\section{Our Test Case: Aventurine Glass}

Aventurine glass, sometimes called Goldstone, is a synthetic substance which is notable for exhibiting the effect of aventurescence (which was named after it) particularly clearly. It is a type of opaque glass with copper crystal inclusions; the inclusions are the deliberate result of a specialised production process. This accounts for its ready availability, the consistent properties of specimens, and the low price. In its most common form it is red, although there are also green or blue versions.

We chose this particular material as the test case for our investigation because it is easy available, strongly shows the properties we are interested in, and - in contrast to many other aventurescent gemstones, which also exhibit several other, prominent but unrelated visual effects - only has this one extraordinary appearance feature. Also, its glittering effect is still phenomenologically identical to that found in real semi-precious gemstones that exhibit aventurescence, such as Sunstone.

\subsection{Properties of Aventurine Glass}

If one examines the appearance of a specimen of Aventurine glass, one notices two key propeties:

- Structure and Colour. Aventurine glass consists of many different particles embedded in glass. Due to the different thickness of the glass layer that coats the individual metallic particles, the light that is reflected from them underlies a varying degree of absorption. Therefore the particles all appear to have different colours, and the stone looks freckled.

- Glittering effect. One distinct feature of the typical aventurescence glitter is that it is orientation dependent. When turned around, certain, clearly distinguishable individual particle facets begin to sparkle. This effect is - at least in principle - present in all surfaces that use a layer of metallic flakes or crystals to obtain their appearance. However, it is not always directly visible; for example, metallic car paints frequently use glitter particles of microscopic size to deliver a smooth finish. In aventurescent gems the particles are much larger and further apart, which leads to the characteristic, individual sparkles found on such stones.

In the following sections we present our experiences on how one can efficiently model these features in a photorealistic rendering system.

All experiments discussed in this paper were done with a physically-based rendering framework that supports unbiased global illumination computations, spectral representations of light, procedural textures, volumetric rendering, and layered surface models [Weidlich and Wilkie 2007].

\section{Modelling Aventurine Glass}

The task of representing such a material can be broken down into several sub-tasks: modelling the particle shape, the particle colour, the glittering effect, and the incorporation of volumetric effects.

\subsection{Brute Force}

It is of course theoretically possible to model an aventurescent material by brute force, i.e. the inclusion of individual crystals as geometric objects within the parent substrate. This kind of approach is obviously neither efficient nor, as we will see, actually necessary, since very good results can be obtained through a suitable approximation that retains much of the physical attributes of the original.

Apart from explicit creation of crystal geometry within the gemstone in question, the next most obvious modelling technique would be the use of suitable subsurface scattering functions. Such an approach yields wonderful results for reasonably homogenous scattering materials [Frisvad et al. 2007]. However, it is not really applicable in this particular case, since the visibly inhomogenous micro-structure of such stones would require a scattering function that exhibits strong local variations - which in turn violates one of the assumptions of most efficient rendering approaches for materials that exhibit sub-surface scattering.

\subsection{Our Approach: A Multi-Layer Surface with Procedural Textures}

After analysing the properties of Aventurine Glass, we decided to use an approach that combines several techniques, and - at least in the case of objects with a dense particle population - eschews actual scattering calculations. Most of the visual complexity of the material is represented through a procedurally textured, multi-layer surface, but we retain the possibility to describe volumetric effects in more transparent specimens of the material.

It should be noted that all aspects of our technique are purely procedural; apart from the colour values used to describe individual material components, and apart from the parameters used to control the texture functions, no other form of input - such as $2 \mathrm{D}$ texture maps - is used.

\subsubsection{Performance}

The performance of the presented technique is consistent with other uses of the procedural shader sub-system within the rendering framework we used. Any increases in rendering time for aventurescent objects compared to plain geometries, were similar to those experienced when rendering other procedurally textured objects, i.e. no performance penalties specific to our approach were observed.

Since the shader sub-system of the rendering toolkit in question is fairly similar in its functionality to those found in other graphics packages (such as RenderMan ${ }^{\circledR}$ ), it is reasonably safe to assume that our technique would work equally well on commercial systems that feature a feature-rich shader language. 


\subsubsection{Verification}

The somewhat ad-hoc nature of our technique is vindicated by the quality of the results that can be obtained with it, and show that a careful analysis of a modelling problem can lead to quite simple solutions that are still highly efficient. We performed verification experiments that attempted to assess the visual fidelity of the results we obtained, and did not find any serious deficiencies. Figure 2 shows one example setup, where we compared a sample sphere made from aventurine glass with a rendered image.

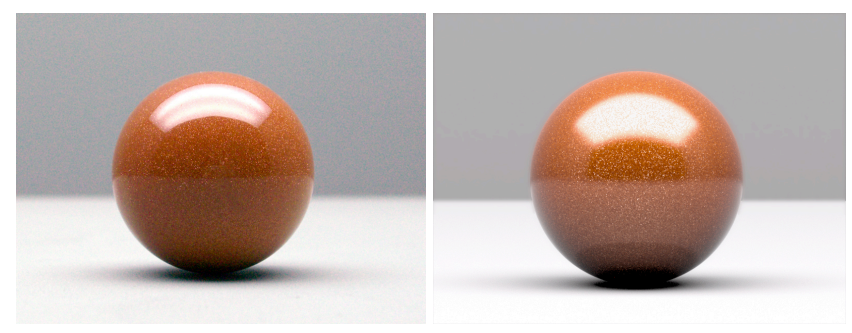

Figure 2: A photograph of a red aventurine glass sphere in a viewing booth (left), and a rendering of a sphere modeled with our technique that was placed in a reasonably similar environment (right). The most prominent difference between the two is the lack of structure in the highlight of the synthetic image, but this is due to the fact that the environments are not completely similar. While the real viewing booth has a diffusor screen between the lights and the actual viewing booth, the fluorescent tubes that provide the lighting are still clearly visible through it, and account for the two stripes in the highlight. Due to limitations in the rendering software used, the ceiling of the virtual viewing booth is just an evenly emissive surface.

\subsubsection{Modelling the Particle Shape}

Since we opted to represent the particle facets as features in a procedural texture on the object surface, the first step is to determine a suitable texture function that yields a realistic-looking surface structure.

As discussed earlier, the glittering particles that are distributed in the transparent or translucent parent material are small crystals, and therefore have smooth polygonal faces that act as tiny mirrors. They are very small, but still large enough and far apart that individual sparkling highlights are caused by single, identifiable crystals.

This means that any texture function that does not yield actual polygonal "glitter elements" will not properly capture the appearance of an aventurescent material. As point in case, we originally experimented with using a Perlin noise function - i.e. a technique with varying but smooth micro-structures - to texture the surface. The reasons for this attempt were the seeming suitability of this function to deliver surfaces with small, irregular structures, and its ready availability in many rendering systems. However, as can be seen in figure 3 , the results are far from satisfactory.

A much more suitable texture function is a Voronoi cell function; this kind of texture leads to many, more or less evenly sized polygonal cells of irregular shape, that can then be appropriately coloured.

While a single Voronoi texture would already look significantly better than a surface based on Perlin noise, the more or less uniform cell size does not resemble the appearance of a real aventurescent stone.
In a real stone, particles that are deeper in the material appear smaller, both because they are further down (for very opaque aventurescent materials, this has a minor to negligible influence, though), and because they are far more likely to be occluded by other particles (this is always relevant).

These seemingly different particle sizes can easily be modeled by using two or more different Voronoi tesselations with unequal cell size that are overlaid over each other, instead of using a single texture for all particles.

Particles that are closer to the surface are generated with a Voronoi function that has bigger cells. In this top-level texture layer (referred to as the "glitter map" (2) in figure 4), only a few of the cells are used, leaving the remainder transparent. This ensures that the lower levels of the layered texture are still visible, and effectively removes the characteristic and somewhat artificial "look" of a single Voronoi pattern.

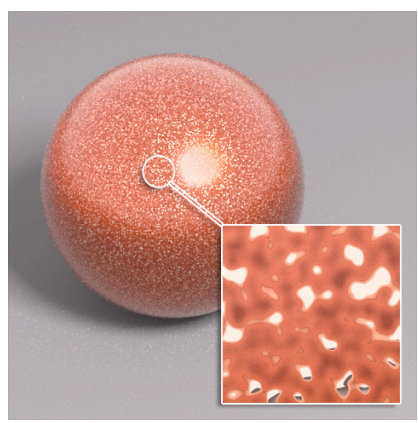

(a)

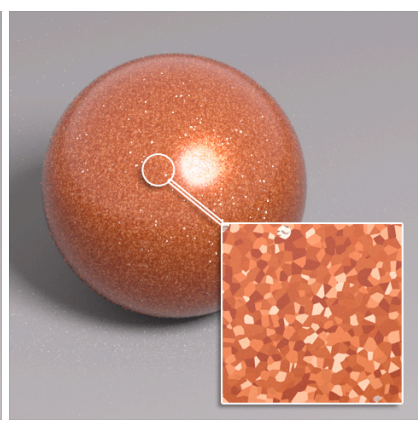

(b)
Figure 3: Spheres with different particle shapes. In the close up it can be clearly seen that the particles generated with Perlin noise are too round and smooth to resemble a real aventurescent material, while the particles generated with the Voronoi cell texture are much more realistic.

\subsubsection{Modelling the Particle Colour}

While the main, transparent substrate of Aventurine glass is, as the name implies, just fairly evenly tinted glass (usually of a mostly transparent, reddish-brown colour), the overall colour of such an object is far from uniform. The reason for this are again the crystal inclusions, which are located at varying depths within the substance.

Crystal particles that are very deep within the glass do not contribute much to the appearance of the stone, since not enough light reaches them to produce any noticeable glitter or colour; all they do is to increase the opacity of the object.

It is the particles in the vicinity of the surface that are responsible for the freckled colour variations that are apparent in such objects.

Capturing this effect is not difficult: in the lower of the two Voronoi tessellation textures (i.e. the one with the smaller cell size, and without the glitter elements discussed later), the cells are coloured so that the bigger cells are filled with less saturated metallic reflectors, while the others are filled with the smaller, more saturated ones. As sketched in element (1) of figure 4, we use a number of predefined tinted, layered surfaces of increasing layer thickness, which are then used to colour the cells of the Voronoi texture. The individual tinted, layered surfaces are similar to those discussed in [Weidlich and Wilkie 2007]. 
In the classification given there, what we use would be a type d) "metal foil" surface - a smooth tinted dielectric layer that covers a smooth metallic substrate. The colour of the tinted layer is the colour of the base material (in our case, the colour of the aventurine glass itself), and the reflectance of the metallic base substrate depends on the metal the particles are made from (in the case of aventurine glass, copper).

As can be seen in element (2) of figure 4, this colouring principle also extends to the larger cells found on the topmost layer. The few cells that are used are covered by a selection of the same kind of metallic layer surfaces, but with even thinner layer thicknesses to yield very de-saturated colours.

\subsubsection{The Glittering Effect}

So far, we have assumed that the cells of the Voronoi texture are all perfectly flat and aligned with the "parent" surface.

However, modelling all the visible particle facets as metallic, polygonal texture elements with normals that are always parallel to the surface normal does not do the appearance of real aventurescent materials justice. Although the particles would have a metallic appearance, no sparkling effects away from the standard surface highlight would be visible, because all particle facets appear to have the same local orientation. In real aventurescent materials, the particles are more or less randomly oriented. See figures $5 \mathrm{a}$ and $5 \mathrm{~b}$ for static images of the difference this makes; the effect is much more striking when comparing animations of a rotating sphere.

To overcome this problem we used a bump map to modify the surface orientation of the large Voronoi cells in the topmost texture layer - the so-called glitter map.

Due to their size, and their assumed proximity to the surface, it makes sense to see these large Voronoi cells as representing entire particles with several visible facets, instead of single particle facets like the cells in the lower texture levels.

The reflections from particles further down in the parent material are strongly attenuated by absorption, so the assumption that one only sees the single, brightest reflecting facet - which is very likely to be oriented towards the surface in this case - is a valid simplification for the lower layer.

For the large facets of the glitter map texture, we use bump mapping to modify the normal vector in a fashion that simulates several small facets that lie within this region. This yields an appearance and sparkling behaviour that is fairly similar to a - computationally much more costly - introduction of real crystal particle geometry at this level.

The exact distribution used for the perturbation of the particle facet normal vectors is a parameter that can be used to control the appearance of the object. We used a function that creates little irregular polygonal "pyramids" over each of the particle facets in the glitter map. The illustration of component (4) in figure 4 shows a small sketch of this "pyramid" bump mapping scheme.

An additional advantage of this kind of cell subdivision is that each of these additional, bump-mapping induced particle facets gives more chances to produce a sparkle than if only the entire top-level polygonal particle facet were to be tilted.

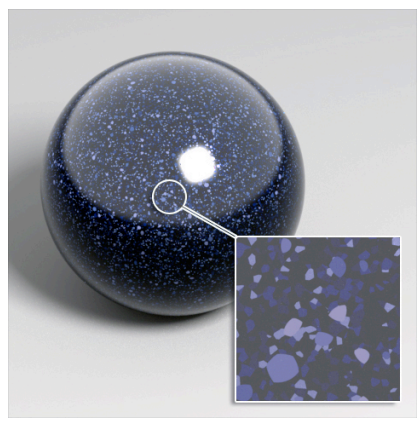

(a)

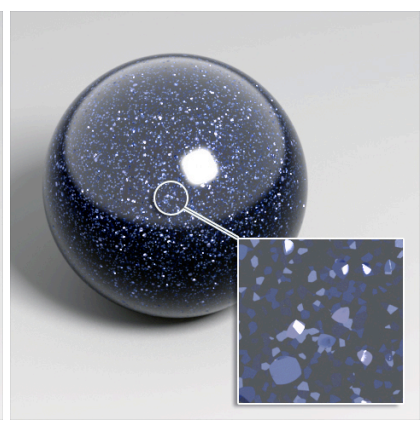

(b)
Figure 5: The same stone, without and with additional bumpmapped particle facets on the topmost layer, i.e. the glitter map facets shown in element (5) of figure 4. The left sphere without the glitter facets sparkles only around the highlight, while the right sphere sparkles also in the area where the surface normal of the sphere tilts away from the light source.

\section{Using the Model for Other Aventures- cent Gemstones}

Although we demonstrated our approach on the test case of opaque aventurine glass, it is not limited to that. With a few parameters changes we can simulate a variety of different aventurescent minerals.

Apart from their colour, the main difference between the individual mineral types are the degree of transparency (which ranges from transparent through translucent to completely opaque) and the distribution and amount of glitter. All three factors can be controlled very easily.

\subsection{Colourisation}

In contrast to Aventurine Glass, which is artificially created through a well-defined process, most aventurescent minerals are the product of complex natural crystallisation processes, and are coloured more or less irregularly.

From a modelling perspective, this just amounts to variations in the base colour of the aventurescent material, though, which is something that can easily be incorporated using standard procedural texturing methods. Examples like the Lavender Lepidolite or the Raspberry Aventurine in figure 6 derive most of the colour aspects of their appearance from normal procedural textures that were chosen to match the real stone in question.

Changing the colour of a stone is mainly a change of the surface map (element (1) in figure 4). Figure 7 gives a rough overview how the appearance of the Lepidolite from figure 6 was done; the majority of the appearance is derived from the surface map in this case, and not the glitter map.

Element (1) is replaced by several surface maps of different resolution; two surface maps, one with more and one with less saturated tiles, are combined with another surface map with much bigger tiles, thus creating a pattern of comparably large alternating dark and bright tiles, which in turn consist of many small tiles. This technique creates a freckled appearance that still shows some global order. The combined surface map is - together with a third, even brighter and more tessellated Voronoi surface map - then used as 


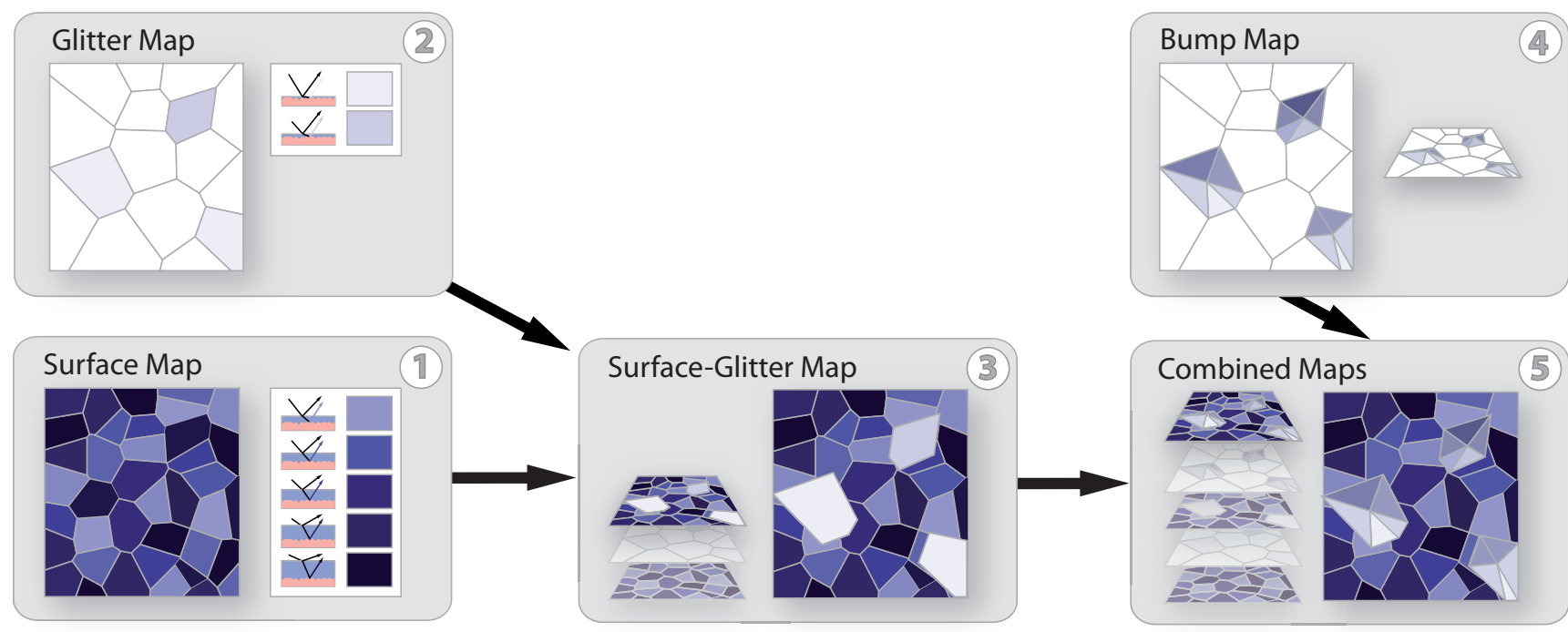

Figure 4: Schematic illustration of the spatial arrangement of the Voronoi cell texture layers. The cells of textures that represent individual particle facets located on a deeper level are smaller (component (1) - the Surface Map), and the texture layer nearer to the top (layer (2) - the Glitter Map) includes fewer and larger cells that are assumed to stand for entire particles. Most of the cells in (2) are completely transparent, with only those responsible for the glitter remaining. Component (4) - the crystal facet bump map - provides sparkle facets for exactly those cells in layer (2) that are not transparent. Note that the varying colour of the bump map facets in this drawing is not due to different types of surface being used, but due to shading effects of the now non-planar surface! The combined result (5) mostly consists of polygonal facets of varying colour intensity, with some of them exhibiting additional sparkling effects due to their modified surface normal.

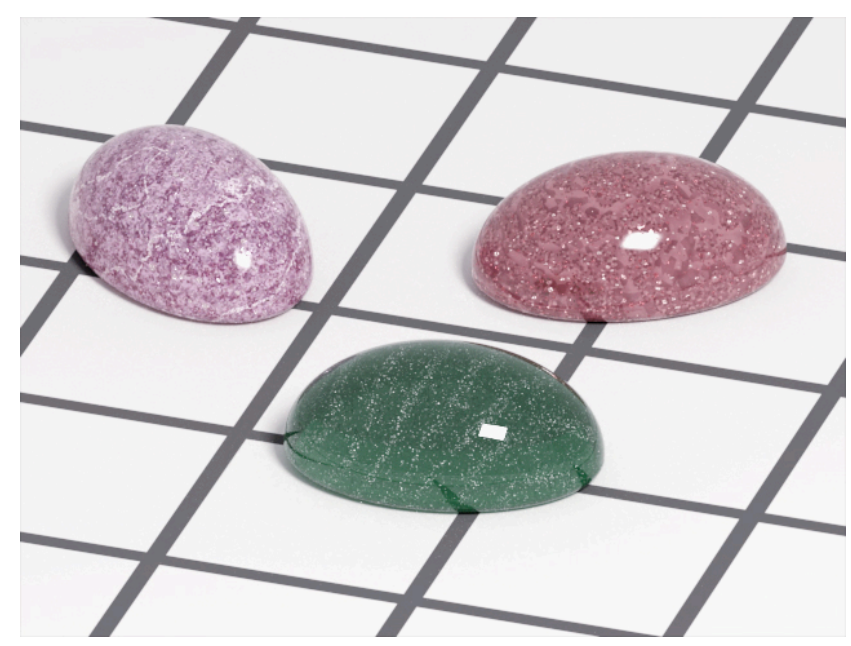

Figure 6: Samples of various almost opaque, aventurescent minerals. While the Lavender Lepidolite (left stone) is so opaque that only a few sparkles are visible, the Raspberry Aventurine (top right) is slightly more transparent and therefore exhibits a much stronger aventurescence effect. The amount of glitter that a gem exhibits is ajusted by modifying the glitter map. Note that the glitter does not have to be uniformly distributed, as we demonstrate with the striped Green Aventurine specimen (middle). input in a marble texture to produce the white veins typical for this mineral. The whole arrangement replaces element (1).

In the case of such strongly structured, opaque base materials, the bottom surface map also contains transparent cells, or, much as in the case of volumetric materials discussed in section 5.3, is omitted altogether in favour of only using a glitter map over the normal, procedural base material texture.

\subsection{Variation of the Glitter}

In addition to the hand-crafted base colour textures of such stones, our approach allows one to add the appropriate amount of glitter to the stone.

Size and amount of glitter can be change in element (2) - the larger the tiles, the bigger is the glitter. By altering the ratio between transparent tiles and tiles that are filled with a metallic surfaces, the amount of glitter can be modified as well. Figure 8 shows a series of stones with decreasing amount of glitter; the fewer elements there are in the glitter map, the less sparkly the stone will appear.

We could also adjust the orientation of the glitter in element (4); this would be useful for materials with particles that are not isotropically distributed.

\subsection{Incorporation of Volumetric Effects}

As described so far, our technique is mainly useful for parent materials that are either comparatively opaque, and/or have a dense population of glitter flakes. For such objects, the effect of aventurescence can be adequately described by a layered surface model alone. 

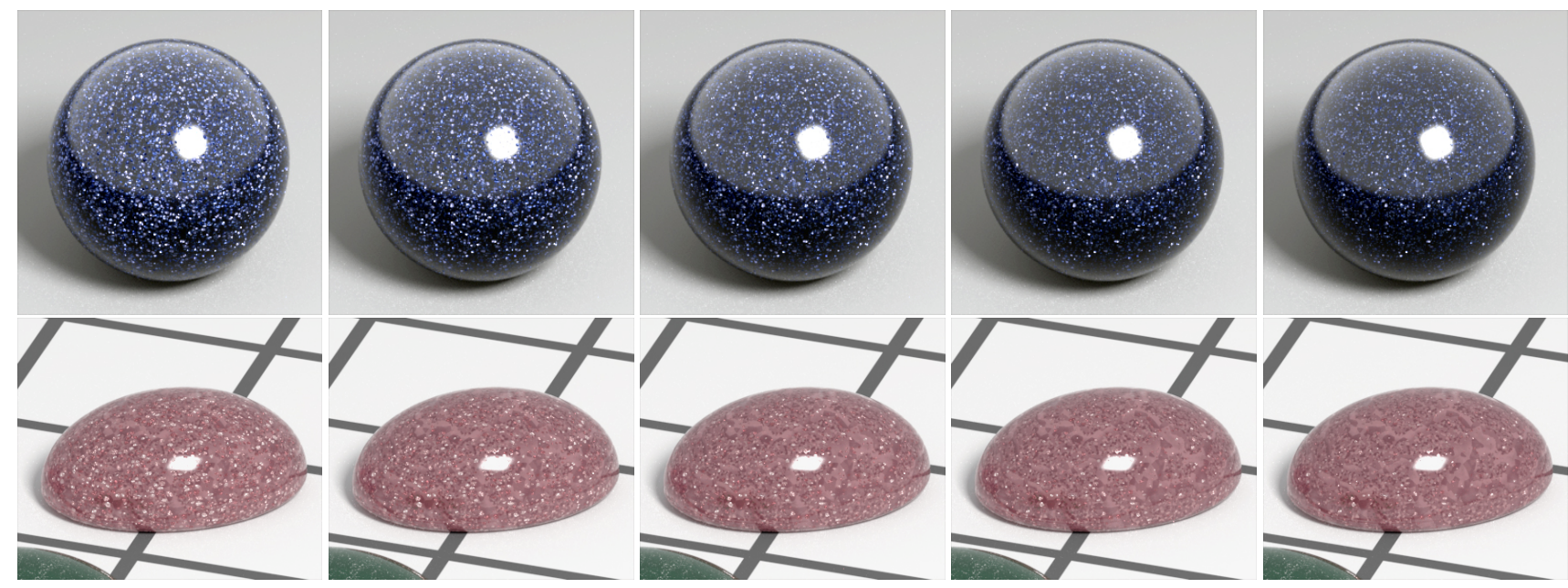

Figure 8: The effect of varying the glitter map density, demonstrated on blue Aventurine Glass (top), and Raspberry Aventurine (bottom). From left to right, the number of non-transparent particle facets in the glitter map decreases, with a corresponding decrease in "sparkliness" of the entire stone. Note that the underlying surface map remains exactly the same in both cases.

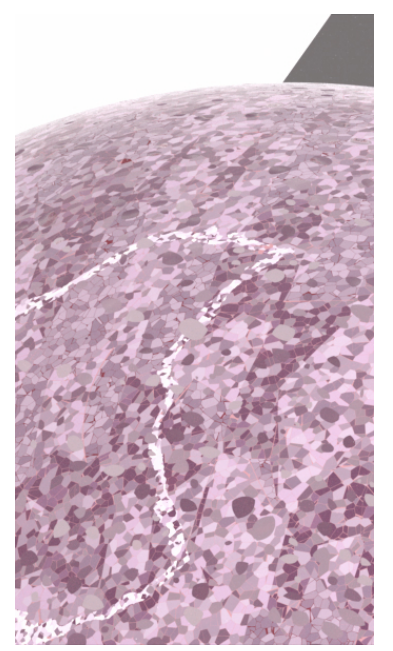

(a)

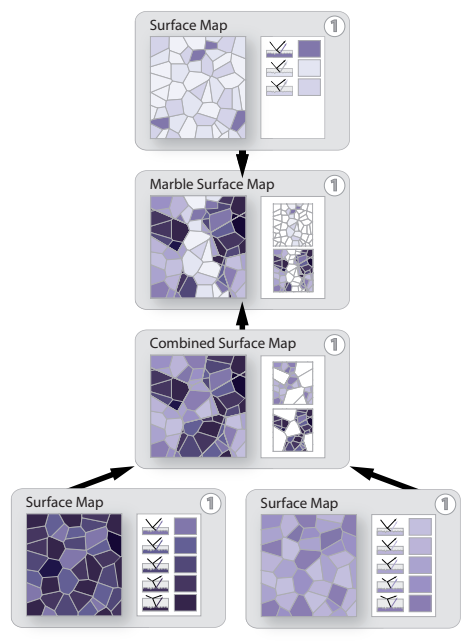

(b)

Figure 7: (a) A close-up of the Lepidolite from figure 6, which reveals that - like in a real stone of this type - the overall appearance of the stone is caused by a complex pattern of polygonal particle facets, which are further influenced by colour changes driven by low-frequency, marble-like patterns within the material. To produce this effect, the simple arrangement of layered surfaces used for Aventurine Glass is replaced by a more complex combination of various surface maps outlined in (b). Each of these maps in turn consists of several layered surface elements, and a procedural marble texture function is used to switch between the individual maps.

Not all aventurescent materials fit these criteria, though. For example, there are several common forms of Sunstone, some of which are quite transparent. Figures 1 and 9 show examples of this material; in its unmodified form, our approach would not be able to handle such translucent, sparkling gemstones.

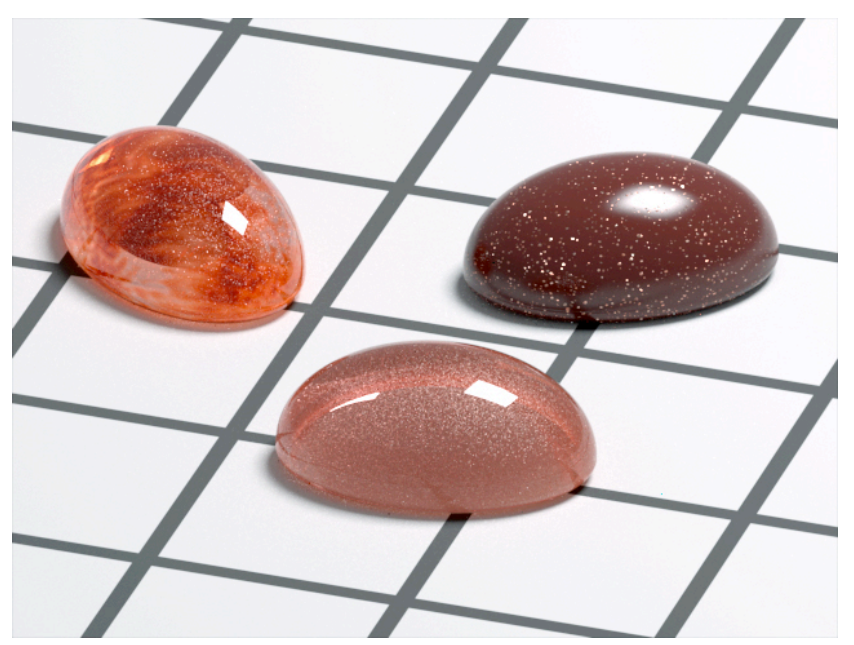

Figure 9: Example renderings of various forms of Sunstone. The metallic glitter and orientation-dependent sheen have made this particular mineral a sought after gemstone. Commercial gems often use a cut similar to these virtual specimens in order to present these properties in an optimal way. Note that only the specimen on the top right is opaque; the other two are translucent, volumetric objects with a glitter map.

The key for an easy extension to such materials are the observations that even in such materials, only the topmost layers of particles contribute to the sparkling effect, and that the remaining translucency properties can be described fairly well by using standard volumetric rendering techniques.

Therefore the only real modifications we had to make in order to achieve results like in figure 9 was to omit the surface map (component (1) in figure 4), and to only use a bump-mapped glitter map. 
This mostly transparent surface was applied to a structured, translucent object that - taken by itself - contains no sparkling components at all, and that was rendered using a standard GI renderer capable of handling scattering materials.

Somewhat surprisingly, the fact that this simplification places all glitter particles on the surface of this object is not visually apparent. This can be explained by two observations: firstly, that in real translucent, aventurescent objects our assertion about only the topmost layers of particles contributing to the overall visual appearance holds, and secondly, that the varied "depth" at which the particle facet colouring described in section 4.2.4 seems to place the individual particles is a good technique to simulate particles that are distributed within the material.

\section{Limitations of Our Approach}

As the results indicate, our modelling technique is capable of efficiently representing several well-known types of aventurescent gemstone with a high degree of realism.

However, we had to make several assumptions in order to derive this technique, and these do not hold for all conceivable aventurescent materials, so it is important to also note where the applicability of the presented technique starts to break down.

The approximation for volumetric materials presented in section 5.3 is only really valid if the parent material is not completely clear, and if a reasonable number of particles is present. Specifically, our technique will not work for small numbers of particles suspended in an otherwise transparent material; in this case, the fact that all sparkling elements are located on the surface would start to be obvious.

Particles that are too large or too small would probably also cause a degradation of model efficiency; the former due to misrepresentation of the appearance, and the latter simply because other techniques start to be much more efficient once individual particles start being so small that they can no longer be discerned. In this case we would recommend to use the model of [Ershov et al. 2001].

\section{Conclusion}

We presented our experiences with physically-oriented, efficient modelling of aventurescence, a very visually appealing property of several types of semi-precious gemstones. Due to its nature, the effect requires careful analysis before an efficient model representation can be found. The solution we used is very probably also applicable to other, similar appearance attributes of gemstones and man-made objects.

\section{A Parameters}

Our approach uses a combination of several well-known but nontrivial techniques to simulate aventurescence. The numerical parameters we used for the demonstrations are strongly dependent on the actual implementation of the various components involved in the simulation, so it would be rather pointless to document them in this paper.

In order to give others a chance to reproduce our results, we instead opt to describe our use of the various components as accurately as possible for each of the demonstrations.

\section{A.1 Aventurine Glass}

Figure 2, 3 and 5 were modelled with the same assembly of surfaces. The surface map has 18 entries of the same surface type a layered surface with a copper base coated with a tinted blue or orange varnish layer, with an index of refraction of 1.53. While the varnish layer is always smooth, the copper base has a varying roughness, that lies between 1 and 10 degrees. The thickness of the layer also varies.

The glitter map has only four entries, and both its base and top are smooth. Compared to the surface map, the layer thickness is rather thin (about one tenth). Glitter and surfaces are not evenly spaced; there are much more transparent cells in the glitter map than filled ones. Please note that the full surface model is only applied to the spheres in figures 2 and $5 \mathrm{~b}$, and the ring in figure 1 .

\section{A.2 Lavender Lepidolite}

The overall modelling of the Lavender Lepidolite in figure 6 is described in section 5.1. The surface map of the dark tiles has 9 entries, but in contrast to the Aventurine glass, most of the surfaces have a Lambertian base of different colour (we used 6 different colours picked from a copy of the NCS colour atlas) and a smooth layer with index of refraction of 1.56. Only one entry has a metallic (in this particular case, silver) base with an average micro-facet slope of 1 degree. The layer above that is smooth and pinkish (similar to the colour of the tiles). The second surface map of the bright tiles is built in a similar fashion, except that the colours of the Lambertian bases are brighter. In this case, we used only 5 different colours. Dark and bright tiles have the same size and are recursively used in another surface map with bigger tiles.

The third surface map for the marble inclusions has only 3 surface entries, which are even lighter in colour than the bright tiles. In order to disrupt the otherwise continuous lightly coloured line, entries from the other tile layers are also used. Please note that the tiles of the marble surface map are much smaller than the dark or bright tiles.

The glitter map is built similar to that of the Aventurine glass with 4 entries. They have a silver base, but otherwise everything remains the same. Only very few entries of the glitter map are filled.

\section{A.3 Raspberry Aventurine}

The Raspberry Aventurine from figure 6 is comparatively easy to build. Three surface maps are used, one with 9 entries and two with two entries each. The surfaces from the first map are like those of the Lavender Lepidolite, a Lambertian base of different colour with a smooth layer (the average micro-facet slope is 1 degree) and one metallic layered surface. Both these layers are smooth. The second and third surface map also contain surfaces with a smooth topmost layer (the IOR is 1.6) over a Lambertian base.

The tiles of the first surface map are much smaller than the tiles of the other two. All three surface maps are combined in another surface map, and are distributed with a marble texture function.

The glitter map is built as in the other cases. The glitter tiles are bigger than the tiles of the first surface map, but not as big as the tiles of the second or third surface map. The distribution of the glitter is even, but again, only few entries of the glitter map are filled. 


\section{A.4 Green Aventurine}

The Green Aventurine in figure 6 has no surface map. The glitter map has 4 entries which all have a silver base surface with an average micro-facet slope of 3 degrees, and a smooth green tinted layer of varying thickness. The transparent cells are also smooth, and have an index of refraction of 1.6. Underneath is a volumetric material that very strongly scatters light.

The stripes of the Green Aventurine are realised with an agate texture function. For the stripes the same glitter map is used in all places. In areas with denser glitter the the glitter map simply contains fewer transparent tiles than in those with less glitter; the shape and size of the cells remains constant.

\section{A.5 Sunstone}

The opaque Sunstone in figure 9 has no surface map either, because it is so opaque that hardly any variation in colour can be seen. Therefore we used the same Torrance-Sparrow surface with a Lambertian base and smooth varnish to fill all transparent tiles of the glitter map. Note that we used the reflection spectrum of a real Sunstone for the colour.

The glitter map again has four entries. The base material of the glitter is copper, the average micro-facet slope is 3 degrees. The varnish has the colour of the Lambertian base and has an index of refraction of 1.58. The average micro-facet slope is 4 degrees. The relation between glitter and non-glitter tiles is the same as for plain Aventurine glass.

The transparent and the translucent stone are created in much the same way as the Green Aventurine. The glitter map has 3 entries, the base material is copper and the average micro-facet slope is 5 degrees. The tiles are tinted with the same colour the stone exhibits. The pink stone exhibits much more glitter, because a large number of cells in glitter map are filled. In contrast to that, the patterned Sunstone exhibits considerably less glitter, but the scattering function is no longer homogeneous. To produce the desired effect we combined three different 3D Perlin functions with different parameters. The index of refraction again is 1.58 for both stones. The same material was used for the necklace in figure 1.

\section{References}

Ershov, S., Khodulev, A., And Kolchin, K. 1999. Simulation of sparkles in metallic paints. In Proceeding of Graphicon, 121-128.

Ershov, S., Kolchin, K., And MyszKowski, K. 2001. Rendering pearlescent appearance based on paint-composition modelling. Comput. Graph. Forum 20, 3, 227-238.

ERshov, S., Ďurikovič, R., Kolchin, K., AND MYSZKOWSKI, K. 2004. Reverse engineering approach to appearance-based design of metallic and pearlescent paints. The Visual Computer 20, 8-9, 586-600.

Frisvad, J. R., Christensen, N. J., And Jensen, H. W. 2007. Computing the scattering properties of participating media using lorenz-mie theory. ACM Trans. Graph. 26, 3, 60.

GuY, S., AND Soler, C. 2004. Graphics gems revisited: fast and physically-based rendering of gemstones. ACM Trans. Graph. $23,3,231-238$.
Sun, Y., Fracchia, F., AND Drew, M. 1999. Rendering the phenomena of volume absorption in homogeneous transparent materials. In Proceeding of the 2nd Annual IASTED International Conference on Computer, 283-288.

Sun, Y., Fracchia, F., AND Drew, M. 2000. Rendering diamonds. In Proceedings of the 11th Western Computer Graphics Symposium (WCGS), 9-15.

Tannenbaum, D. C., Tannenbaum, P., and Wozny, M. J. 1994. Polarization and birefringency considerations in rendering. In SIGGRAPH '94: Proceedings of the 21 st annual conference on Computer graphics and interactive techniques, ACM, New York, NY, USA, 221-222.

Thomas, S. W. 1986. Dispersive refraction in ray tracing. The Visual Computer 2, 1 (Jan.), 3-8.

Ďurikovič, R., Kolchin, K., AND Ershov, S. 2002. Rendering of japanese artcraft. In Proceedings of the EUROGRAPHICS short presentations, 131-138.

ĎURIKOVIČ, R. 2002. Explicit method of sparkling effect simulation. Journal of Three Dimensional Images 16, 4 (Jan), 96-100.

WeIDLICH, A., AND WILKIE, A. 2007. Arbitrarily layered microfacet surfaces. In GRAPHITE '07: Proceedings of the 5th international conference on Computer graphics and interactive techniques in Australia and Southeast Asia, 978-1-59593-9128, New York, NY, USA, 171-178.

Wilkie, A., Tobler, R. F., and Purgathofer, W. 2001. Combined rendering of polarization and fluorescence effects. In Proceedings of the 12th Eurographics Workshop on Rendering Techniques, Springer-Verlag, London, UK, 197-204.

WolfF, L. B., AND KURLANDER, D. 1990. Ray tracing with polarization parameters. IEEE Computer Graphics \& Applications 10, 6 (Nov.), 44-55.

YuAn, Y., Kunit, T. L., InAmoto, N., AND Sun, L. 1988. Gemstone fire: adaptive dispersive ray tracing of polyhedrons. The Visual Computer 4, 5, 259-270. 